\title{
Notes pour servir à la rédaction d'une histoire de seigneurie
}

\section{Raymond Douville}

Volume 3, numéro 3, décembre 1949

URI : https://id.erudit.org/iderudit/801574ar

DOI : https://doi.org/10.7202/801574ar

Aller au sommaire du numéro

Éditeur(s)

Institut d'histoire de l'Amérique française

ISSN

0035-2357 (imprimé)

1492-1383 (numérique)

Découvrir la revue

Citer cet article

Douville, R. (1949). Notes pour servir à la rédaction d'une histoire de seigneurie. Revue d'histoire de l'Amérique française, 3(3), 325-332.

https://doi.org/10.7202/801574ar d'utilisation que vous pouvez consulter en ligne.

https://apropos.erudit.org/fr/usagers/politique-dutilisation/ 


\section{NOTES POUR SERVIR À LA RÉDACTION D'UNE HISTOIRE DE SEIGNEURIE}

Sous ce titre, je voudrais tout simplement énumérer quelques impressions et opinions personnelles, recueillies au cours d'incursions effectuées dans le passé de deux ou trois seigneuries de chez nous, pour relater leurs origines et me familiariser avec leurs fondateurs et leurs pionniers.

Il existe d'excellentes études qui font autorité sur la façonou les façons - de rédiger une monographie seigneuriale. Il importe d'en connaître l'essentiel, et même de les bien assimiler, car la monographie est une branche de l'histoire, et l'histoire, personne n'en peut douter ici, est une science trop importante pour que chacun la traite selon son caprice ou sa fantaisie. Cependant, à trop suivre les règles établies, on risque d'être terne, monotone, et de manquer de vie, point pourtant bien essentiel à qui veut reconstruire, même ressusciter le passé. Car ressusciter veut bien dire: ramener de la mort à la vie, rendre vivant ce qui ne l'était plus.

De tous les domaines de l'histoire, il n'en est pas un, je crois, qui nous permette de ranimer le passé comme la monographie. Le sujet, dans sa diversité, s'y prête admirablement. Et il est tout aussi possible pour l'historien que pour le peintre de reproduire avec exactitude toutes les nuances d'une fresque du passé, en la rendant personnelle tant par le style que par le coloris des détails.

$\mathrm{Au}$ risque de passer pour fantaisiste, je placerais au premier rang des raisons qui peuvent inciter un écrivain à s'intéresser à une seigneurie, l'amour du coin de terre qu'il veut étudier. Cet amour lui sera une source de satisfaction constante au milieu de tous les déboires qu'il ne manquera pas de rencontrer au cours de ses recherches, et il peut sourdre en lui de différentes façons: soit qu'il s'agisse de sa paroisse natale ou du lieu d'arrivée de son premier ancêtre, ou encore que les exigences de sa profession l'obligent à y passer sa vie, comme c'est le cas, par exemple, pour un curé, un médecin, un notaire ou un insti- 
tuteur. Toutes ces personnes ont plus d'avantages qu'un étranger, même si elles ne sont pas historiens de métier, pour écrire une histoire à caractère personnel, vivante, précise, pleine de détails pittoresques et précis sur le manoir seigneurial, les familles, le sol, les vieilles maisons, l'emplacement des premières concessions, le développement économique, etc.

Cette affection naturelle pour les gens et les choses dont on recré la vie est un aliment précieux pour celui qui veut écrire une histoire fortement documentée et toute de couleur locale; elle permettra de s'attarder dans les méandres du passé comme si on accomplissait un pèlerinage, et facilitera le moyen de se plonger dans l'atmosphère véritable de l'époque.

Voilà pour la partie sentimentale, la partie inspiration.

Dans le domaine pratique, il importe d'abord pour l'historien de se faire une idée aussi précise que possible du régime seigneurial d'autrefois. Cette façon d'octroyer des terres, originaire de l'ancienne France, comportait chez nous des caractéristiques particulières. D'excellents travaux ont été publiés sur le sujet, dont le plus récent et le plus complet est celui de Mtre Victor Morin: "Seigneurs et censitaires, castes disparues", " puissante évocation du régime seigneurial canadien, étude indispensable à qui veut entreprendre d'écrire l'histoire d'une seigneurie.

D'une façon générale, il est préférable de présenter au lecteur, en un chapitre condensé, la situation géographique de la seigneurie, avant que de faire connaître celui qui en fut le premier seigneur. $\mathrm{Ce}$ qui permettra de relater les menus faits peu connus dont cet endroit a pu être le thêatre avant l'établissement officiel. La plupart des seigneuries établies le long du Saint-Laurent ont une pré-histoire intéressante, grâce aux séjours qu'ont pu y faire les tribus sauvages, les missionnaires, les trafiquants de fourrures et même quelques colons solitaires.

Une biographie du premier seigneur aurait ensuite sa place. Relater ses origines, ses titres de noblesse et sa carrière militaire, s'il y a lieu, son caractère, en somme toutes les caractéristiques qui pourront démontrer la tendance que prendra par la suite la seigneurie.

1. Victor Morin, Seigneurs et censitaires, castes disparues. (Montréal, Les Editions des Dix, 1941). 
Puis commence pour l'historien le véritable travail local, qui est de tirer des archives tous les détails possibles sur les personnes et les événements qui, de près ou de loin, touchent au sujet que l'on veut traiter.

Travail qui peut paraître ingrat, qui rebute bien des novices, surtout quand les manuscrits sont d'une lecture difficile; qui peut paraitre monotone aussi, quand les textes semblent à peu près tous identiques, par exemple dans les contrats de concession. Mais l'essentiel est de ne rien laisser au hasard, de tout lire, de tout noter, et surtout de tout retenir. Car une phrase cachée dans un texte apparemment sans importance peut être la seule à fournir la clef d'un problème de date ou d'emplacement. L'ingratitude de ce travail de recherches patientes est vite compensée chez le chercheur par la passion quil'anime peu à peu et l'amour de ses personnages.

On ne saurait attacher trop d'importance à cette méticuleuse précision d'information. Mieux vaut ne rien écrire que de rester dans le vague. Les historiens de la grande histoire connaissent bien la satisfaction que leur procurent les informations puisées dans une monographie qu'ils savent être exacte, parce qu'un renseignement puisé dans un travail quj manque de précision les oblige à une double recherche: dans cet ouvrage d'abord, parce qu'ils espèrent $y$ trouver ce qu'ils cherchent, et aller à la source même de la documentation pour s'assurer de son exactitude. Donc, double perte de temps. Si l'historien se contente de faire sien ce renseignement inexact sans le vérifier, il s'en rend complice et le couvre de son autorité, ce qui est encore plus grave.

Le monographe doit donc se faire un devoir d'être honnête envers les historiens qui utiliseront le fruit de ses recherches. Il doit être honnête aussi envers le public, envers ses lecteurs, et particulièrement envers les familles dont il étudie l'ascendance. Car souvent les seuls renseignements que certaines familles peuvent obtenir sur leurs ancêtres se trouvent dans cette monographie, et l'auteur est responsable envers ces familles de la sûreté de son information.

Un problème se pose parfois: doit-on rapporter dans une monographie certains événéments malheureux qui pourraient jeter du déshonneur ou du discrédit sur une famille? En général, le soin de signaler ou d'ignorer ces événements doit être laissé au bon jugement de celui qui les rapporte. S'ils n'ont pas de réelle valeur historique, inu- 
tile de les citer. Si ces événements ont une portée qui dépasse l'intérêt particulier, l'historien ne peut les taire. A lui d'agir avec la discrétion qui s'impose.

Ecrire une monographie seigneuriale, c'est tout d'abord faire un travail de petite histoire. Or, la petite histoire est faite de petits détails. Et ces petits détails, ce sont les premiers seigneurs et les premiers colons qui nous les apportent. Autour du manoir seigneurial naissent l'une après l'autre les coutumes locales qui font le charme de la vie d'autrefois. L'historien ne peut les négliger, de même qu'il pourra relater la façon dont s'exerçait la moyenne et basse justice, ce qui fera connaître à la fois le caractère du seigneur et celui de ses censitaires.

Où trouver la documentation?

Il existe deux sources principales d'information: une source locale, par les registres de paroisse et les papiers de famille; l'autre, générale, se trouve dans les greffes de notaires et aux archives provinciales et fédérales surtout. Les inventaires après décès des pionniers d'une paroisse sont une source précieuse, de même que les contrats de mariage et les actes de donation.

Avant que de dresser le plan de son travail, il importe, je crois, d'épuiser autant que possible toutes ces sources d'information. Et c'est ici qu'un monographe peut et doit se révéler original. La masse de documentation recueillie devra influencer et diriger la marche du récit. Voici pourquoi.

Le véritable fondateur d'une paroisse, souvent, c'est non pas le seigneur, mais un simple colon, un défricheur, un attaché au sol, qui avait du bon sens, le sens de l'organisation, un bon jugement, de la ténacité. C'est ce véritable pionnier qu'il faut découvrir, et, quand on l'a découvert, le mettre en vedette. Plus que le seigneur, psychologiquement, il est suivi, il est écouté des autres, il donne l'exemple, et c'est à lui que la paroisse doit son développement.

Historiquement parlant, les seigneuries se suivent et ne se ressemblent pas. Voici un exemple qui m'a particulièrement frappé, lorsque je ramassais les matériaux pour l'histoire des premiers seigneurs et colons de Ste-Anne de la Pérade. Du côté de Québec, Ste-Anne a pour voisine la paroisse de Grondines (ou St-Charles-des-Roches). Du côté de Trois-Rivières, c'est Batiscan, qui elle-même touche à la seigneurie de Champlain. Or, ces quatre seigneuries voisines, colonies à la même époque, soit entre 1665 et 1672, ont des origines entièrement 
différentes et ont été colonisées pour des motifs absolument dissemblables.

Ste-Anne fut d'abord un poste de traite. Et c'est pour activer son commerce plus que pour le défrichement que le premier seigneur, Michel Gamelain, y amena des colons. Donc, il importait au seigneur d'avoir des recrues disposées à courir les bois plutôt qu'à labourer le sol. En 1672, la seigneurie passa à $M$. de Lanaudière, un militaire qui ne fit pratiquement rien pour le développement de sa seigneurie, et à $M$. de Suève, un célibataire qui semble avoir complètement manqué de sens d'organisation. Donc ici les seigneurs ne comptent pas comme fondateurs proprement dits. La seigneurie de Grondines avait été octroyée en 1639 aux Sœurs Hospitalières de Québec, et ce n'est que vers 1670 qu'elles réussirent à y établir des colons. La seigneurie fut vendue à Jacques Aubert, qui s'en désintéressa, puis en troisième lieu à Louis Amelain, qui lui donna un vigoureux essor vers 1700 , soit environ 60 ans après qu'elle eut été octroyée aux Religieuses. La seigneurie de Batiscan appartenait aux Pères Jésuites qui, de leur mission du Cap de la Madeleine, y dirigèrent des colons choisis par eux. La seigneurie prospéra vite. A Champlain, M. de la Touche obtint la seigneurie en 1665, il s'y attacha, et tout de suite les colons sérieux affluèrent.

Donc, quatre seigneuries voisines, quatre débuts différents. A Grondines, la seigneurie languit, faute de tête dirigeante. A SteAnne, ce sont les colons eux-mêmes qui dirigent le défrichement. A Batiscan, les Jésuites dirigent la seigneurie de leur mission du Cap et en font un succès. Il n'y a qu'à Champlain que nous trouvons sur place un seigneur véritable et conscient de son rôle, de ses devoirs et de ses responsabilités.

J'ai cité cet exemple pour démontrer que si tous les monographes s'employaient à bien marquer les détails caractéristiques des débuts, l'historien pourrait ensuite comparer et juger, à l'aide de ces humbles travaux, les causes diverses qui ont amené la fondation et le développement des seigneuries. La plupart des manuels d'histoire nous enseignent que la vie paroissiale se greffait sur le manoir seigneurial. En fait, ce n'est pas toujours exact, et surtout il est faux de prétendre de façon générale que le seigneur était l'âme de la paroisse. Un travail fouille sur chacune des seigneuries d'autrefois nous apprendrait qu'en réalité le vénérable fondateur de la seigneurie de Boucherville, Pierre 
Boucher, demeuré le modèle sympathique des seigneurs conscients de leur rôle, eut malheureusement peu d'imitateurs. Faire le partage de la gloire qui revient aux seigneurs et du mérite des premiers missionnaires résidents et des véritables colons pionniers d'une seigneurie est un devoir auquel les historiens ne peuvent se soustraire.

C'est pourquoi je suis d'avis que la relation des débuts d'une seigneurie offre la plupart du temps la matière suffisante d'un ouvrage complet par lui-même. Pour une seigneurie octroyée vers 1665 ou 1672, on peut prendre comme limite le recencement de 1681 . Ou encore, ce qui est préférable, les dénombrements seigneuriaux effectués à diverses époques jusque vers 1725 et dans lesquels on trouve généralement la liste de tous les colons, l'endroit de leur concession et le nombre d'arpents de terre en culture.

Ce travail complet sur les origines offre plusieurs avantages. Tout d'abord, il montre à l'œuvre le premier seigneur et les premiers pionniers, leurs difficultés, leurs succès et leur part réciproque dans le développement de la seigneurie. Il permet aussi à l'historien de s'attarder à un travail anecdotique et biographique plus élaboré sur ces fondateurs. Ces notules historiques et généalogiques peuvent être données en appendice, pour ne pas alourdir le récit, soit par ordre alphabétique, soit par ordre chronologique d'arrivée dans la seigneurie. $\mathrm{Si}$ le monographe en a le gout, et surtout le loisir, il pourra donner la liste complète des colons qui ont passé dans la seigneurie, même ceux qui n'y ont pas fait souche. Ce travail a son utilité, soit pour les historiens, soit pour les généalogistes. De toute façon, il ne sera pas perdu, car aucun détail en histoire n'est inutile.

Quelques-uns me reprocheront peut-être d'accorder trop d'importance à cette période des débuts, de trop tenir compte des détails. Je crois pourtant que les facilités de plus en plus grandes qui nous sont fournies aujourd'hui de nous documenter obligent à ce travail de recherches minutieuses, si on veut donner à son travail une réelle valeur historique. Travail d'archiviste, sans doute, mais tout historien doit être avant tout un archiviste.

Quand le monographe se sera ainsi formé la main - et le caractère - à bien dépouiller les textes qui couvrent les premiers trente ou quarante ans d'une seigneurie, rédiger avec méthode la suite des événements, le progrès et le démembrement de la seigneurie, lui paraîtra facile. Le temps me manque pour esquisser un projet complet. D'ail- 
leurs, d'excellents modèles ont été tracés par des experts dans ce domaine. M. l'abbé Ivanhoë Caron, dans une étude à la Semaine d'Histoire du Canada en 1925, a suggéré des plans utiles, tant pour les seigneuries que pour les paroisses de fondation ancienne ou récente. On y pourra référer avec avantage. Mais ce qui est mieux, des historiens de valeur se sont attachés à écrire des monographies seigneuriales qui sont des guides d'utile voisinage. Il y a par exemple cette œuvre devenue presque classique: "L'Histoire de la Seigneurie de Lauzon" de M. Edmond Roy, et celle, toute différente dans sa conception parce que le sujet le réclamait, du R.P. Thomas-M. Charland, 'L'Histoire de Saint-François-du-Lac". Il en est une autre pour laquelle j'ai personnellement beaucoup d'estime, et qui est peu connue. Elle s'intitule humblement "Les Annales de Lotbinière" 2 et a pour auteur un ancien curé de l'endroit, l'abbé Louis-L. Paradis. On y trouve beaucoup de renseignements sur les origines, une ordonnance claire, un style vivant, et même un sens critique allant jusqu'au scrupule, si on en juge par la façon dont l'abbé Paradis parle de lui-même et de ses vingt-cinq ans de direction pastorale. Voici par exemple en quels termes il jugea bon de se faire connaître lui-même à la postérité: "D'une manière générale, écrit-il à la page 334 de sa monographie, la population de nos campagnes aime peu les changements de curé, et c'est avec regret que Lotbjnière vit partir l'abbé Demers. Son successeur en office fut l'abbé Louis-L. Paradis... D'un physique peu avantageux, qu'accentuait une démarche singulière, tout son extérieur annonçait une santé débile, et les bonnes gens de Lotbinière en augurèrent que son séjour au milieu d'eux ne serait pas bien long... Réservé et peu expansif, il inspirait un certain embarras à ceux qui l'approchaient, et pendant un long gouvernement de vingt-cinq ans, tout en jouissant de l'estime et du respect des paroissiens, l'abbé Paradis ne fut jamais très populaire..."

Voilà un bel exemple de franchise et d'honnêteté. Il mérite d'être signalé, et tous ceux qui écrivent des monographies, seigneuriales ou paroissiales, devraient demander à la Providence de leur accorder un peu de ce sens de l'équilibre et de sincérité dont elle avait si bien gratifié M. l'abbé Paradis.

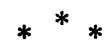

2. Abbé Louis-L. Paradis, Les Annales de Lotbinière (1672-1933) Imprimé aux ateliers de l'Action Catholique, (Québec, 1933), 444 pages, avec portrait et plans. 
D'une façon générale, le régime seigneurial, qu'on a tant décrié parfois, était le plus logique pour l'époque, et M. Victor Morin, dans l'ouvrage déjà mentionné, avait raison d'écrire que "ceux qui ont blâmé la France d'avoir introduit ce régime au Canada, n'ont pas réfléchi que c'était alors un système économique et social consacré par des siècles d'existence et qu'en l'appliquant à ce pays, on lui a fait subir des modifications profondes, pour l'adapter à ses besoins et aux conditions de vie de ses habitants. Les colons venus de France y étaient habitués; ceux d'entre eux que le métier des armes unissait à leurs officiers par le lien puissant des dangers courus ensemble, étaient tout préparés à collaborer entre eux, non seulement pour leur défense mutuelle contre un ennemi dix fois supérieur en nombre, mais encore pour le développement de leur nouvelle patrie. C'est ce qui explique comment la Nouvelle France a pu résister si longtemps aux tentatives de conquête de ses voisins, en dépit de son infériorité économique".

Ces justes réflexions de M. Morin disent assez combien il est important de dépouiller et d'ordonner tous les documents généraux et familiaux qui ont trait à chacune des seigneuries d'autrefois. Lorsqu l'histoire de chacune aura été écrite, par des historiens consciencieux, avec toutes leurs particularités, il n'est pas exageré de dire qu'elles formeront la base de l'histoire du régime français au Canada.

Raymond Douville Trois-Rivières, P.Q. 